\title{
Saúde mental do estudante universitário: um relato de experiência de extensão no período de pandemia
}

\author{
University student mental health: a report of extension experience in the pandemic period \\ Salud mental de estudiantes universitarios: un informe de experiencia de extensión en el período de
} pandemia

Recebido: 23/11/2021 | Revisado: 02/12/2021 | Aceito: 15/12/2021 | Publicado: 22/12/2021

\author{
Luana Foroni Andrade \\ ORCID: https://orcid.org/0000-0003-2765-1535 \\ Universidade Federal de Sergipe, Brasil \\ E-mail: luanaforoni@gmail.com \\ Raphaela Schiassi Hernandes \\ ORCID: https://orcid.org/0000-0002-9290-1003 \\ Universidade Federal de Sergipe, Brasil \\ E-mail: rapha_to@hotmail.com \\ Danillo de Menezes Araújo \\ ORCID: https://orcid.org/0000-0001-7410-8354 \\ Centro Especializado de Reabilitação do tipo IV, Brasil \\ E-mail: terapeutadanillo@gmail.com \\ Halley Ferraro Oliveira \\ ORCID: https://orcid.org/0000-0003-0123-7395 \\ Universidade Federal de Sergipe, Brasil \\ E-mail: halleyoliveira62@gmail.com
}

\begin{abstract}
Resumo
A pesquisa objetiva relatar a experiência e o impacto na saúde mental de estudantes universitários participantes de um projeto de extensão durante o período de pandemia. O estudo foi desenvolvido a partir da coleta de dados quantitativos e qualitativos por meio de um formulário online com questões abertas e fechadas que convidava o participante a analisar sua saúde mental antes e durante o período suspensão do calendário acadêmico, com oferta de atividades remotas, devido à crise sanitária causada pelo coronavírus. Os resultados apontam que houve impacto importante na saúde mental da maioria dos estudantes. Durante a pandemia, 54,5\% dos participantes declararam que na maior parte do tempo consideram a saúde mental regular e 36,4\% disseram que a maior parte do tempo era ruim. Após a participação no curso, 54,5\% passou a declarar que na maior parte do tempo sua saúde mental era boa, com queda sobre aqueles que consideravam a maior parte do tempo regular, 27,3\%. Espera-se que essa experiência corrobore com ações futuras dessa natureza, visando o bem-estar e a saúde de estudantes universitários.
\end{abstract}

Palavras-chave: Saúde mental; Saúde do estudante; COVID-19.

\begin{abstract}
The research aims to report the experience and impact on the mental health of university students participating in an extension project during the pandemic period. The study was developed from the collection of quantitative and qualitative data through an online form with open and closed questions that invited the participant to analyze their mental health before and during the suspension period from the academic calendar, with the offer of remote activities, due to the health crisis caused by the coronavirus. The results show that there was an important impact on the mental health of most students. During the pandemic, $54.5 \%$ of the participants declared that most of the time they considered their mental health to be regular and $36.4 \%$ said that most of the time was bad. After participating in the course, $54.5 \%$ went on to declare that their mental health was good most of the time, with a drop over those who considered most of the regular time, $27.3 \%$. It is hoped that this experience will corroborate future actions of this nature, aiming at the well-being and health of university students.
\end{abstract}

Keywords: Mental health; Student health; COVID-19.

\section{Resumen}

La investigación tiene como objetivo reportar la experiencia y el impacto en la salud mental de los estudiantes universitarios que participan en un proyecto de extensión durante el período pandémico. El estudio se desarrolló a partir de la recolección de datos cuantitativos y cualitativos a través de un formulario en línea con preguntas abiertas y cerradas que invitaban al participante a analizar su salud mental antes y durante el período de suspensión del calendario académico, con la oferta de actividades a distancia, debido a la crisis sanitaria provocada por el 
coronavirus. Los resultados muestran que hubo un impacto importante en la salud mental de la mayoría de los estudiantes. Durante la pandemia, el 54,5\% de los participantes declaró que la mayoría de las veces consideraba que su salud mental era regular y el $36,4 \%$ dijo que la mayor parte del tiempo era mala. Tras participar en el curso, el $54,5 \%$ pasó a declarar que su salud mental era buena la mayor parte del tiempo, con un descenso sobre los que consideraban la mayor parte del tiempo regular, un $27,3 \%$. Se espera que esta experiencia se corrobore con acciones futuras de esta naturaleza, orientadas al bienestar y la salud de los estudiantes universitarios.

Palabras clave: Salud mental; Salud del estudiante; COVID-19.

\section{Introdução}

Estudo recente com a população de estudantes universitários revelou a alta prevalência de Transtornos Mentais Comuns (TMC), conhecido como transtornos psiquiátricos menores, com presença de insônia, diminuição de concentração, impacto na memória, dificuldade para tomada de decisões, sintomas como fadiga, irritação, sensação de inutilidade e sintomas somáticos (Preto et al., 2020).

A presença de sofrimento psíquico tem sido relacionada a aspectos ligados à vida acadêmica entre universitários, com predominância de fatores como as séries do curso e a percepção negativa do ambiente, sendo estes fatores de risco possíveis de intervenção e modificação para aumento do bem-estar entre os alunos e vivências no contexto educacional mais positivas. Aspectos como hábitos prejudiciais à saúde e problemas de saúde têm sido associados à presença de TMC, enquanto que o apoio social tem sido associado a um menor sofrimento psíquico (Graner \& Cerqueira, 2019). Estes aspectos levantam a necessidade de atenção de gestores, profissionais e pesquisadores para o desenvolvimento de propostas e intervenções voltadas ao cuidado e apoio dos estudantes.

No início do ano de 2020, a Organização Mundial da Saúde (OMS) declarou que o surto da doença causada pelo novo coronavírus (COVID-19) consistia em uma emergência de saúde pública de importância internacional, conduzindo ações diante do mais alto nível de alerta da Organização, em conformidade ao regulamento sanitário internacional. Em março, a COVID-19, doença infecciosa causada pelo novo coronavírus, passou a ser caracterizada como uma pandemia, sendo que até 14 de agosto de 2020 os dados confirmavam no mundo 20.730.456 casos de COVID-19 e 751.154 mortes (OPAS, 2020; UNA-SUS, 2020). Dados da OMS, em novembro de 2021, mencionavam o impacto da pandemia com o alcance de 5.047 .652 mortes em todo o mundo (OMS, 2021), 2.309.394 só nas Américas (OPAS, 2021), sendo que no Brasil os óbitos confirmados na mesma data mostravam 609.573 mortes (MS/DATASUS, 2021).

Diante da crise sanitária a Organização Pan-Americana da Saúde, junto à Organização Mundial de Saúde tem disponibilizado uma série de ferramentas e medidas para auxiliar os governos na tomada de decisão sobre medidas não farmacológicas, como endurecimento ou afrouxamento das medidas de distanciamento social (OPAS, 2020). Os impactos em diversos setores como econômico, político e educacional criaram a necessidade de intervenções imediatas. Uma das medidas foi a suspensão dos calendários acadêmicos nas universidades.

Uma pesquisa realizada em Portugal, no início da Pandemia, teve por objetivo analisar os níveis de ansiedade, depressão e estresse em estudantes universitários com comparação entre duas amostras distintas: uma nos anos entre 2018 e 2019 e outra amostra recolhida nos oito dias após a suspensão das aulas no ensino universitário e o decreto que instituiu o estado de emergência em Portugal em março de 2020. Os resultados deste estudo mostraram níveis elevados na amostra de 2020, em quarentena, comparada a anterior, com impacto negativo nos aspectos psicológicos, alertando para a necessidade de traçar estratégias de prevenção e intervenção, buscando estilos de vida saudáveis, manutenção de redes sociais de apoio através das tecnologias de informação e comunicação, promover uma postura mais criativa ou de mobilização de recursos ou estratégias anteriores para lidar com situações adversas, programas de promoção de competências sociais e emocionais, bem como ações para minimizar os efeitos traumáticos decorrentes desta pandemia (Maia \& Dias, 2020). 
A exploração dessa temática torna-se urgente, uma vez que o sofrimento psíquico tem sido relatado na literatura como um tema de fundamental importância na população de estudantes universitários. O relato de experiência a partir dos dados coletados proporcionará contribuição para discussões da experiência formativa e do cuidado em saúde mental ofertado a esse público, além de contribuir para o campo científico.

Diante das reflexões expostas, foi criado um curso remoto voltado à Saúde Mental de estudantes universitários durante o período de pandemia, sendo a presente pesquisa desenvolvida com o intuito de analisar e relatar a experiência e os impactos na saúde mental dos participantes do curso.

\section{Metodologia}

Trata-se de um relato de experiência a partir da realização de um curso de extensão voltado à saúde mental de estudantes universitários durante o período da pandemia. Para atingirmos o objetivo e analisar a experiência vivida durante o curso, a pesquisa foi delineada a partir da coleta de dados quantitativos (números brutos e porcentagens a partir de alternativas de respostas ou escalas de 0 a 10) e qualitativos (questão aberta).

Devido ao período de crise sanitária da Pandemia ocasionada pelo novo Coronavírus com consequentes adaptações nas atividades de ensino, pesquisa e extensão, esta pesquisa foi realizada de maneira remota por meio de formulários online pelo googleforms, aplicada com participantes de um curso que foi planejado visando o acompanhamento e acolhida de estudantes universitários, com encontros semanais em plataforma virtual com duração de duas horas, sendo oferecidas informações e estratégias para manejo de situação de estresse e ansiedade, orientações e atividades inventivas para ressignificar o cotidiano e as situações de crise enfrentadas. Os encontros aconteciam semanalmente de maneira remota, por meio de uma plataforma digital (google meet), com duração de aproximadamente duas horas. No total foram 14 encontros, sendo que o primeiro foi de apresentação, o último de fechamento, um dos encontros foi assíncrono para leitura de materiais e vídeos e os outros onze encontros foram síncronos, sendo que cada um apresentava um tema a ser vivenciado por meio de palestra e/ou vivência de acordo com o professor convidado. Os temas foram: Equilíbrio entre Trabalho e Lazer; Ops! Caiu a Conexão! Quando comunicar é uma barreira; O que fazer com uma folha de papel; Sentidos - Cotidianos e sensações; Comunicação não violenta; O lado bom dos coisas ruins; Cotidianos e Corpos inventivos; A arte como forma de Autoconhecimento; O que é felicidade? Xô Estresse! Como situações estressantes podem modificar conexões neurais e estruturas cerebrais e o que fazer para se manter saudável? e A viagem mais importante de sua vida.

A população do estudo refere-se a todos os inscritos no curso de extensão "Pega Leve: Saúde Mental de estudantes universitários", criado pelo Departamento de Terapia Ocupacional da Universidade Federal de Sergipe, Campus Lagarto. A amostra foi composta pelos participantes que atingiram a carga horária de no mínimo $75 \%$ da carga horária total prevista do curso e aceitaram participar do estudo respondendo o formulário online elaborado pelos coordenadores do estudo. Os critérios de inclusão foram: estudantes universitários de uma universidade federal no nordeste brasileiro, estudantes da UFS que participaram do curso de extensão "Pega Leve: Saúde Mental de estudantes universitários"; participantes do curso com 18 anos de idade ou mais.

O instrumento de pesquisa trata-se de um questionário online com questões abertas e fechadas disponíveis a partir da plataforma googleforms criado pelos coordenadores do curso para avaliar a experiência e impacto do projeto de extensão na saúde mental dos participantes antes e durante a pandemia. O instrumento continha questões desde: "Como você avaliaria sua saúde mental antes da pandemia?", “Como você avaliaria sua Saúde Mental durante a Pandemia?"e "Após sua participação no curso Pega Leve, como você avaliaria sua Saúde Mental durante a Pandemia?”, sendo as opções de resposta a maior parte do tempo ruim, a maior parte do tempo regular, a maior parte do tempo boa ou a maior parte do tempo muito boa. Ainda foi perguntado "Como você avaliaria o impacto do curso Pega Leve para melhoria da sua Saúde Mental" a partir de uma escala de 
0 a 10 , sendo 0 nenhum impacto e 10 total impacto. Ao final foi perguntado com opção de resposta aberta " Como você definiria sua experiência no curso?"

Os dados quantitativos foram transferidos para uma planilha do excel e analisados a partir do software Statistical Package for the Social Sciences - SPSS versão 22.1. Para análise dos dados, na fase descritiva, foram usados números brutos, frequências simples e porcentagens. Para análise dos dados qualitativos presentes nas questões abertas que convidam o participante a narrar sua percepção, foi realizada análise de conteúdo.

A pesquisa possui aprovação em Comitê de Ética e Pesquisa (CEP), CAEE 37251320.6.0000.5546, parecer n0.385.139. Os participantes do curso de extensão foram contatados via e-mail e convidados a participar do estudo.

\section{Resultados}

Dos 29 alunos inscritos no curso, 22 aceitaram participar desta pesquisa. O perfil de alunos revelado apresenta que, no momento da pesquisa, $14(63,6 \%)$ estudantes cursavam terapia ocupacional, dois cursavam enfermagem $(\mathrm{n}=2,9,1 \%)$, um cursava odontologia $(4,5 \%)$, um cursava nutrição $(4,5 \%)$, um cursava fisioterapia $(4,5 \%)$, um cursava matemática $(4,5 \%)$, um cursava pedagogia $(4,5 \%)$, e um cursava engenharia de produção $(4,5 \%)$.

No item que avaliava a saúde mental antes da pandemia na percepção dos próprios estudantes, os resultados apontaram que 11 participantes (50\%) declararam que sua saúde mental era boa a maior parte do tempo. Oito (36,4\%) participantes disseram que era regular na maior parte do tempo. Dois $(9,1 \%)$ disseram que a maior parte do tempo era ruim e 1 $(4,5 \%)$ que a maior parte do tempo era muito boa.

Durante a pandemia, a variável sofreu alterações, sendo que 54,5\% dos participantes passaram a declarar que na maior parte do tempo consideram a saúde mental regular, e 36,4\% disseram que a maior parte do tempo era ruim.

Com a participação no curso, as variáveis sofrem nova alteração trazendo resultados mais positivos com a experiência, sendo que 54,5\% passaram a declarar que na maior parte do tempo era boa, com queda sobre aqueles que consideravam a maior parte do tempo regular, 27,3\%, ver Figura 1.

O impacto do curso para desenvolver habilidades de enfrentamento de crise e maior manejo ao estresse, melhorando a percepção da saúde mental também foi evidenciado quando questionado sobre o impacto e as mudanças percebidas, sendo que das 22 respostas, um participante trouxe que não percebeu impactos e dois apontaram sentimentos negativos à Pandemia. Os demais trouxeram em suas respostas a percepção de benefícios como: melhor compreensão de si mesmo e do outro; dar valor ao momento presente; não se sentir sozinho; dar valor as pequenas coisas e ressignificar o cotidiano; mudança de olhar pessimista para otimista; ter novos pontos de vista; ser grato; diminuição da ansiedade; sentir-se mais preparado e adaptado para as aulas remotas; reinventar o momento que está vivendo; melhora da autoestima; maior equilíbrio emocional; mudar a forma de encarar as situações; melhora na saúde física, emocional e mental nas esferas familiar, acadêmica e pessoal; atitudes mais positivas diante de situações de mudanças e estresse; maior identificação com os outros universitários; lidar com a falta de controle sobre algumas situações; respeitar o próprio tempo; maior habilidade de adaptação ao mundo virtual. 
Figura 1 - Gráfico sobre a percepção da saúde mental nos três períodos analisados (antes da pandemia, durante a pandemia e após o curso), 2020 - Lagarto/SE.

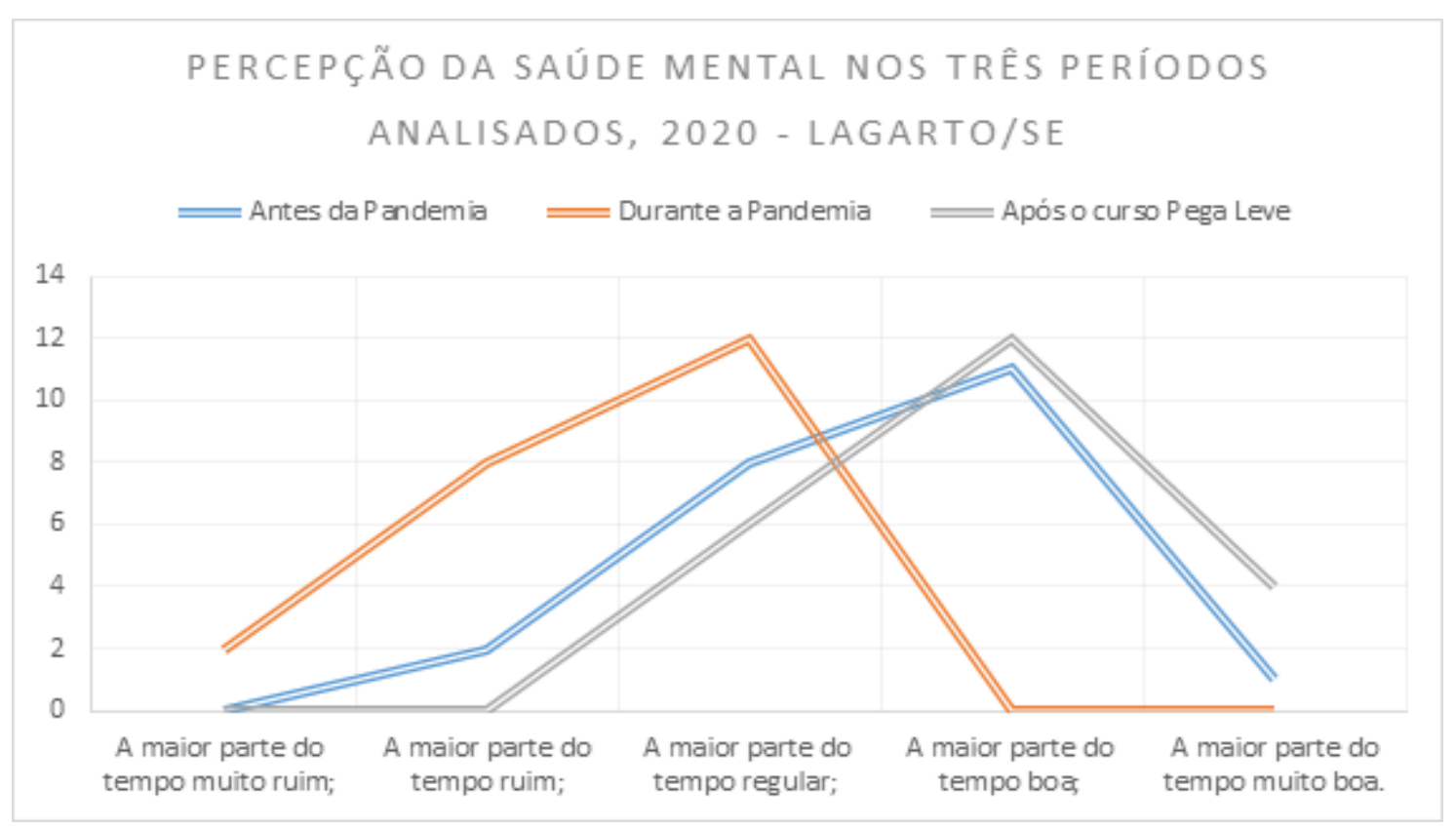

Fonte: Autores.

Quando questionados quanto a como definiriam sua experiência no curso, todas as respostas traziam aspectos positivos com palavras como essencial, gratificante, transformadora ou proveitosa. Sendo o curso definido com palavras como enriquecedor, necessário, extraordinário, esclarecedor, incrível, inovador.

\section{Discussão}

O cenário de crise pandêmica apresentou aos docentes desafios importantes relacionados à mudança do ensino para o ambiente virtual. Foi necessário repensar o aprendizado e o manuseio das tecnologias, informação e comunicação na modalidade remota, buscando não só motivar e estimular os discentes para construção do conhecimento, mas também garantir a finalização do ano letivo (Silva et al., 2020).

As consequências para a saúde mental de todos os envolvidos, principalmente discentes, foram inúmeras, sendo necessário articular e propor ações voltadas ao cuidado a este público. A presente pesquisa propôs analisar quantitativamente e qualitativamente os efeitos da participação de um grupo de estudantes universitários em um curso de extensão de acolhimento e promoção da saúde mental. As categorias de análise das percepções e narrativas, trazidas na questão aberta do instrumento, serão apresentadas a seguir como: acolhimento e suporte, prevenção ao adoecimento psíquico, e cuidado e apoio, somadas a outros achados na literatura que coadunam também com os resultados quantitativos apresentados neste artigo.

\section{Acolhimento e Suporte}

O acolhimento e suporte aparecem como estratégias fundamentais para continuidade do vínculo e possibilidade de auxiliar os estudantes no enfrentamento da crise. Percepções com discursos nesta direção foram observados na análise qualitativa nos dados coletados na questão aberta quanto à experiência com o curso:

"Um espaço de autocuidado, gratidão e que me fez refletir sobre o período de pandemia como um momento de novas descobertas. "(Participante 3) 
"Diferente e transformadora, estava habituada com outros cursos que focam mais em aspectos didáticos" (Participante 11)

"Mudei muito a minha percepção quanto a realidade e me ajudou muito a ressignificar os meus dias aproveitando cada momento do hoje ao invés que aguardar que tudo volte ao normal, já que temos um futuro incerto." (Participante 14)

Antes mesmo da pandemia, estudos já demonstravam a importância em dar atenção e propor intervenções nas universidades pensando na saúde mental dos graduandos. A exemplo da pesquisa de Veloso e colaboradores (2019), que encontrou a expressiva prevalência de $22 \%$ sobre ideação suicida entre os acadêmicos, sobretudo entre homens solteiros e com vínculo empregatício. Ainda foi destacado que destes, 90,3\% faziam uso de bebida alcoólica, 54,8\% de tabaco e 54,8\% de outras drogas, sendo que ter sido vítima de bullying aumentou em 9,55 vezes e já ter cometido tentativa de suicídio em 243 vezes.

Lacerda (2016) aponta sobre a possibilidade de determinadas situações se somarem as vulnerabilidades sociais e emocionais gerando sofrimento psíquico, o que juntamente com as demandas advindas da universidade poderiam desencadear ou até mesmo agravar problemas de saúde física e mental. Por isso, as Universidades podem desenvolver um papel fundamental no cuidado à saúde mental dos estudantes, a exemplo da elaboração de diretrizes e ações de enfrentamento do adoecimento psíquico. Inclusive, é comum que Instituições de Ensino Superior brasileiras atualmente possuam algum tipo de atividade de acolhimento, a partir do entendimento de que ações como essas possam promover integração e cuidado com o universitário, principalmente em tempos como esses vivenciados durante a pandemia.

\section{Prevenção ao adoecimento psíquico}

Um recente ensaio clínico investigou a relação entre saúde mental e a pandemia por Covid-19, encontrando prevalência de depressão e ansiedade de 50,7\% e 44,7\% respectivamente (Liu et al., 2020). Salientando que a literatura mostra que, no caso de pessoas que já apresentam algum antecedente de adoecimento psíquico prévio, a exemplo da ansiedade, os efeitos psicopatológicos associados ao isolamento social são agravados, o que pode ocasionar um aumento na incidência de estresse pós-traumático (Brooks et al., 2020).

Um dos participantes do curso deixa claro a preocupação e o medo de ter uma crise durante a pandemia, destacando a importância de ter participado do curso:

“Fundamental para evitar que eu entrasse em surto psíquico” (Participante 19).

Refletindo sobre fatores que podem ocasionar maior impacto na saúde e desenvolvimento de adoecimento e sofrimento psíquico, chamamos a atenção para aspectos como gênero. No contexto universitário, o sexo feminino tem sido descrito como um fator de risco importante para o aparecimento de estresse e transtornos mentais, o que pode ser justificado pelos múltiplos papéis exercidos pela mulher na sociedade, assim como pelas alterações hormonais que por vezes influenciam em seu humor (Lima \& Brito, 2018). Por isso, o gênero pode e deve ser um fator observado em estudos futuros, pós pandemia.

Ressalta-se ainda a importância de inserir nessa discussão a situação de estudantes que cursam a faculdade em municípios distantes e/ou afastado do seu convívio familiar, já que de acordo com algumas análises, discentes que visitam seus 
familiares com baixa frequência no mês estão propensos a desenvolverem maiores transtornos psíquicos e fazem parte de um grupo cuja prevalência foi acima de 50\% para transtornos mentais comuns (Santos et al., 2017).

As discussões sobre estes aspectos no momento atual, quanto aos impactos negativos da pandemia sobre a saúde mental dos estudantes, extrapolam o âmbito nacional. Em Portugal foi comparado níveis de ansiedade, depressão e estresse pré-pandemia - 2018 e 2019 - e na pandemia com universitários e os resultados demonstraram um aumento significativo de perturbação psicológica (Maia \& Dias, 2020). O que está de acordo com os achados do presente estudo, onde antes da pandemia apenas $9,1 \%$ da amostra considerava sua saúde mental ruim na maior parte do tempo, contra $36,4 \%$ com essa percepção durante a pandemia. O estudo desenvolvido por Zaharieva (2020) também encontrou aumento significativo de casos envolvendo depressão e estresse associados principalmente às incertezas vivenciadas, impactos negativos sobre a saúde pública, aumento da vulnerabilidade da saúde das pessoas e enfraquecimento da sociedade de um modo geral durante a pandemia.

No Brasil, um estudo transversal, realizado no início de 2020, investigou a saúde mental (ansiedade, depressão e estresse) em estudantes universitários durante o estágio inicial da quarentena no Brasil, onde os participantes responderam uma pesquisa online sobre a percepção da COVID19, quarentena, traços de personalidade, ideação suicida e saúde mental; participaram 1957 estudantes e pontuações de saúde mental mais baixas foram associadas a um pior impacto do COVID-19 na vida do entrevistado, maior neuroticismo e maior ideação suicida (Teodoro et al., 2021).

Os impactos provenientes da quarentena sobre a saúde mental são tão devastadores que podem deixar sequelas maiores até mesmo que o número de mortes durante a pandemia. Contribui para isso o cenário caótico, onde os sistemas de saúde podem entrar em colapso a qualquer momento, a longa duração de isolamento social, sem perspectiva de finalização, ambos impactando consideravelmente na saúde mental (Brooks et al., 2020).

Outro estudo, de Mota e colaboradores (2020), desenvolvido com 275 estudantes universitários, apontou que, no segundo mês de isolamento social, 58,5\% da amostra apresentou intenso sofrimento psíquico, com alta taxa de prevalência de TMC. Sobre as formas de enfrentamento que utilizaram para lidar com o isolamento social, a estratégia de fuga e esquiva foi a mais utilizada $(34,5 \%)$. Outras estratégias referidas foram as de autocontrole $(35,6 \%)$, de suporte social $(32,7 \%)$ e de afastamento $(31,5 \%)$. Sendo assim, com exceção da estratégia de suporte social, as demais foram voltadas para o distanciamento emocional da situação, principalmente por aqueles participantes com sofrimento psíquico intenso. Diferentemente dos universitários com possível ausência de TMC, que utilizaram mais frequentemente as estratégias de enfrentamento baseadas na reavaliação positiva e na resolução de problemas, objetivando extrair significado de suas experiências pessoais, novas aprendizagens e mobilização de recursos para maior adaptação.

\section{Cuidado e apoio}

Como estratégia ao enfrentamento da pandemia e proteção da saúde mental, recomenda-se a validação de sentimentos de tristeza, ansiedade ou confusão desencadeados pelo excesso de informações. Assim como incentivo à manutenção de hábitos saudáveis, redes de apoio através das tecnologias e postura criativa ou de mobilização de recursos e estratégias para lidar com situações adversas (Maia et al., 2020). Os resultados desta pesquisa evidenciam importante impacto das ações de cuidado propostas no curso, que buscavam essa validação e estratégias de apoio e manejo com a situação, em que, após a participação dos estudantes no curso, mais da metade da amostra descreveu estados de boa saúde mental após o curso, com narrativas como:

"Percebi impactos em minha saúde mental, física e emocional. Em algumas esferas da minha vida, como na: familiar, acadêmica, pessoal, etc.” (Participante 17) 
"Consigo perceber que a minha atitude mudou para melhor diante de mudanças e situações de stress. Estou conseguindo ter uma visão equilibrada de min, e das minhas falhas e limitações” (Participante 18)

"Me ajudou primeiramente a organizar a semana mentalmente, como também a descansar minha mente ao me permitir pensar em outras coisas pelo menos durante as horas do curso, ao invés de só pensar no caos atual” (Participante 19)

“Quando eu me sentia sozinho, olhava para o curso e tinha uma identificação com os outros universitários... Eu não estava passando por aquele misto de conflitos internos sozinho... Acho que uma rede de apoio foi criada..." (participante 20)

“Respeitar o meu tempo e aprender a lidar com o fato de não ter controle sobre as coisas (Participante 21)

Com objetivos semelhantes, Braga e Santos (2021) explanam em sua pesquisa sobre uma série de ações realizadas com o intuito de contribuir para o bem-estar emocional dos discentes durante o período do ensino remoto emergencial, dentre elas: plantão vídeo chamada, acolhimento via chat, serviço especializado de acompanhamento para alunos com deficiência e cartilha para professores com orientações sobre saúde mental. Importante destacar que muitos alunos acabaram sendo excluídos do ensino remoto por não terem condições de acompanhar as aulas devido ao fato de não possuírem equipamento adequado para essa finalidade. O que cabe atenção e proposições de alcance a este público no futuro.

Nesse sentido, os resultados deste estudo, somados a literatura chamam a atenção para a necessidade de as Universidades desenvolverem estratégias e espaços que promovam acolhimento, identificação e espaço de partilha (seja em ambiente remoto, e quando possível presencial) para e entre os universitários, tendo em vista os benefícios que agregam sobre a saúde mental dos estudantes, como pode ser observado em um dos relatos desta pesquisa:

“Antes do curso eu tinha uma visão negativa a respeito de tudo e pensamentos sombrios me cercavam. Após o curso comecei a enxergar tudo com um novo olhar e voltei a ser a pessoa otimista de sempre”. (Participante 6)

Como limitação do estudo, podemos destacar o alcance de um número pequeno de participantes, desejando que futuros projetos possam ter maior alcance, vide o impacto relatado como positivo na saúde mental dos estudantes. Destaca-se que o curso, de que se tratou esse estudo, contou com a inscrição de 29 estudantes, e cabe aqui questionar se esse número não poderia ter sido mais expressivo, e se houve para isso a existência de alguma barreira ou agente dificultador, a exemplo do acesso à internet. Para Castioni et al. (2021), embora a parcela de estudantes de graduação sem acesso domiciliar à internet seja pequena, a exclusão digital não só reflete como reforça desigualdades. Já que os estudantes mais afetados são aqueles que já se encontram em desvantagens de oportunidades devido à condições econômicas e sociais piores do que as de alunos com acesso ao ensino remoto Necessário ressaltar que a quantidade de estudantes sem acesso pode ser maior durante a pandemia, afinal, existem alunos que precisam mudar de cidade e sair de suas casas para poder estudar, sendo de se esperar que alguns tenham regressado à seus domicílios de origem - que podem não ter as mesmas condições de acesso à internet. E assim, a dificuldade em estudar durante o período da pandemia pode implicar em ampliação da desigualdade no futuro. 


\section{Considerações Finais}

Os resultados revelam o impacto da COVID-19 na saúde mental dos estudantes universitários pesquisados, trazendo o efeito positivo na maioria dos participantes da experiência narrada, a partir da oferta de um curso de extensão para acolhida e promoção da saúde mental. O presente artigo contribui com pesquisas recentes, apresentando a importância de iniciativas com proposições de estratégias para acolhida e suporte aos estudantes em situações de crise.

\section{Referências}

Braga, S. B. \& Santos, T. F. A. M. (2021). A permanência dos estudantes no ensino superior em tempos de pandemia: uma análise a partir das ações de assistência estudantil da Universidade Federal do Pará. REPOD - Revista Educação e Políticas em Debate, 10(3), 1090-1106, https://doi.org/10.14393/REPOD-v10n3a2021-62374.

Brooks, S. K., Webster, R. K., Smith, L. E., Woodland, L., Wessely, S., Greenberg, N., \& Rubin, G. J. (2020). The psychological impact of quarantine and how to reduce it: rapid review of the evidence. The Lancet, 395, 912-920.

Castioni, R., Melo, A. A. S., Nascimento, P. M. \& Ramos, D. L. (2021). Universidades federais na pandemia da Covid-19: acesso discente à internet e ensino remoto emergencial*. Ensaio: avaliação e políticas públicas em Educação, 29(111), 399-419, https://doi.org/10.1590/S0104-40362021002903108

Graner, K. M. \& Cerqueira, A. T. A. R. (2019). Revisão integrativa: sofrimento psíquico em estudantes universitários e fatores associados. Ciência e saúde coletiva, 24(4). https://doi.org/10.1590/1413-81232018244.09692017

Lacerda, A. N. (2016). Indícios de estresse, ansiedade e depressão em estudantes universitários. (monografia). Universidade de Brasília.

Lima, J. K. A. \& Brito, A. P. A. (2018). Desgaste e sofrimento psíquico em estudantes de medicina: uma revisão sistemática. XVII SEPA, 17. Disponível em: http://www.revistas.unifacs.br/index.php/sepa.

Liu, S., Yang, L., Zhang, C., Xiang, Y. T., Liu, Z., Hu, S., \&Zhang, B. (2020) Online mental health services in China during the COVID-19 outbreak. Lancet Psychiatry, 7(4). DOI: https://doi.org/10.1016/S2215-0366(20)30077-8.

Maia, B. R. \& Dias, P. C. (2020). Ansiedade, depressão e estresse em estudantes universitários: o impacto da COVID-19. Estudos de Psicologia (Campinas), 37, e200067. http://dx.doi.org/10.1590/1982-0275202037e200067.

Ministério da Saúde. (MS) (2021). DATASUS. COVID-19 Painel Coronavírus. https://covid.saude.gov.br/

Mota, D. C. B., Silva, Y. V., Costa, T. A. F., Aguiar, M. H. C., Marques, M. E. M. \& Monaquezi, R. M. (2020). Saúde mental e uso de internet por estudantes universitários: estratégias de enfrentamento no contexto da COVID-19. Ciência \& Saúde Coletiva, 26(6):2159-2170. 10.1590/1413-81232021266.44142020.

Organização Mundial da Saúde (OMS). (2021). Weekly operational update on COVID-19 - 8 https://www.who.int/publications/m/item/weekly-operationalupdate-on-covid-19---8-november-2021

Organização Pan-Americana da Saúde (OPAS). (2020). Folha informativa COVID-19 - Brasil. https://www.paho.org/pt/covid19.

Organização Pan-Americana da Saúde (OPAS). (2021). COVID-19 Region of the Americas Update. 3 PM WDC. https://www.paho.org/en/documents/pahodaily-covid-19-update-8-november-2021

Preto, V. A., Fernandes, J. M., Silva, L.P., Reis, J. O. L., Sousa, B. O. P., Pereira, S. S., \&Cardoso, L. (2020). Transtornos Mentais Comuns, Estresse e Autoestima em universitários da área da saúde no último ano. Research, Society and Development, 9(8). <https://doi.org/10.33448/rsd-v9i8.6362>.

Santos, L. S., Ribeiro, Í. J. S., Boery, E. N \& Boery, R. N. S. O. (2017). Qualidade de vida e transtornos mentais comuns em estudantes de medicina. Cogitare Enfermagem, 22(4).

Silva, A. F., Estrela, F. M., Lima, N. S. \& Abreu, C.T. A. (2020). Saúde mental de docentes universitários em tempos de pandemia. Physis: Revista de Saúde Coletiva, 30(2). https://doi.org/10.1590/S0103-73312020300216.

Teodoro, M. L. M., Alvares-Teodoro, J., Peixoto, C. B., Pereira, E. G., Diniz, M. L. N., Freitas, S. K. P., \&Mansur-Alves, M. (2021). Saúde mental em estudantes universitários durante a pandemia de Covid-19. REFACS, 9(2), 372-82. 10.18554/refacs.v9i2.5409.

Universidade Aberta do SUS (UNA-SUS). (2020) Organização Mundial de Saúde declara pandemia do novo Coronavírus. Ministério da Saúde. Fundação Oswaldo Cruz (FIOCRUZ). https://www.unasus.gov.br/noticia/organizacao-mundial-de-saude-declara-pandemia-de-coronavirus

Veloso, L. U. P., Lima, C. L. S., Sales, J. C. S., Monteiro, C. F. S., Gonçalves, A. M. S. \& Silva Jro , F. J. G. (2019). Ideação suicida em universitários da área da saúde: prevalência e fatores associados. Revista Gaúcha de Enfermagem, 40.

Zaharieva, R. (2020). The Danger of Social Isolation During a Pandemic [Internet]. European Public Health Alliance. https://epha.org/the-dangers-of-socialisolation-during-a-pandemic/. 